\title{
Widely used, commercially available Chlamydia pneumoniae antigen contaminated with mycoplasma
}

\author{
R. P. VERKOOYEN, M. SIJMONS, E. FRIES*, A. VAN BELKUM and H. A. VERBRUGH \\ Departments of Clinical Microbiology and*Virology, Academic Hospital Dijkzigt, Rotterdam, The Netherlands
}

\begin{abstract}
Mycoplasma contamination was detected in a widely used commercially available Chlamydia pneumoniae antigen preparation. Contamination was studied with a mycoplasma group-specific 16S rRNA polymerase chain reaction (PCR) and sequence analysis. Several lots of the purified $C$. pneumoniae antigen from the Washington Research Foundation appeared to be contaminated with the same Mycoplasma species, which appeared to be closely related to $M$. arginini. Antigen slides prepared for the detection of chlamydia antibodies by MRL Diagnosties were contaminated with the same Mycoplasma sp. Chlamydia antigen slides from Labsystems OY and two chlamydia complement fixation reagents (Virion International Distribution Ltd and Behring Werke) were not contaminated. It is concluded that commercially available $C$. pneumoniae antigens may contain mycoplasma antigens as well. Although the impact of such mycoplasma contamination on the results of chlamydia serology may not be significant, routine screening of all antigen preparations obtained by tissue culture before their distribution and use is recommended.
\end{abstract}

\section{Introduction}

Serodiagnosis of Chlamydia pneumoniae, C. trachomatis and $C$. psittaci infection is usually based on complement fixation tests (CF), micro-immunofluorescence tests (MIF) or enzyme-immunoassay (EIA) [1-3]. The antigens used in these tests are purified from chlamydia-infected cell cultures. Worldwide, many cells used for cell culture are contaminated with mycoplasma [4]. As interpretation difficulties arose with the MIF for the detection of $C$. pneumoniae-specific antibodies due to non-specific background fluorescence, the mycoplasma contamination of these commercially available chlamydia antigens was investigated with a mycoplasma group-specific 16S rRNA PCR. Sequence analysis was done to identify the species found by PCR.

\section{Materials and methods}

\section{Chlamydia antigens}

Purified C. pneumoniae elementary bodies were obtained from the Washington Research Foundation

Received 23 July 1996; revised version accepted 4 Oct. 1996. Corresponding author: Dr R. P. Verkooyen.
(WRF; Seattle, WA, USA; lot numbers 6, 7 and 17). Slides coated with chlamydia antigen were obtained from MRL Diagnostics, Cypress, CA, USA, and from Labsystems OY, Helsinki, Finland (lot numbers R047994 and 83 PB 1, respectively). These slides are marketed for the specific purpose of serological diagnosis of chlamydial infection. C. psittaci complement fixation (CF) reagents were obtained from Virion International Distribution Ltd, Chan, Switzerland and from Behring Werke, Marburg, Germany (lot numbers 293616.08 and 413588B, respectively).

\section{Mycoplasma PCR}

Chlamydia antigen slides from MRL Diagnostics and from Labsystems OY were washed as described by Gilroy et al. [5]. Briefly, three antigen slides were used for each isolation. Sterile distilled water $(50 \mu 1 / \mathrm{slide})$ was added to the slide with an aerosol-resistant tip. The surface was washed and scraped with the tip to dislodge the material and suspend it in the water. Purified $C$. pneumoniae antigen (c. $10^{9}$ organisms $/ \mathrm{ml}$ ) and $C$. psittaci $\mathrm{CF}$ antigen were diluted 1 in 100 and 1 in 10 with sterile distilled water, respectively. Diluted antigen suspension $(100 \mu 1)$ or chlamydial antigen recovered from slides $(100 \mu 1)$ were pretreated as described by Boom et al. [6]. 
Mycoplasma DNA was amplified by PCR and analysis of the amplified samples was performed as described by van Kuppeveld et al. [7]. A mycoplasma groupspecific primer set was used, which amplifies a 280-bp fragment with the following primers: forward primer GPO-3 (5'-GGGAGCAAACAGGATTAGATACCCT-3') and reverse primer MGSO (5'-TGCACCATCTGTCACTCTGTTAACCTC- $\left.3^{\prime}\right)$. PCR products $(40 \mu 1)$ were analysed by electrophoresis on an agarose $2 \%$ gel stained with ethidium bromide. Southern blot hybridisation was performed with $\left[\gamma-{ }^{32} \mathrm{P}\right] \mathrm{ATP}-\mathrm{end}-$ labelled internal oligonucleotide GPO-2 (5'-CTTAAAGGAATTGACGGGAAC CCG-3'). The sensitivity of the genus-specific mycoplasma PCR with Mycoplasma pneumoniae was $c$, one cfu [8]. All chlamydia antigen preparations were also amplified with a chlamydia-specific PCR to monitor the DNA isolation. $C$. pneumoniae-containing antigens were amplified by a $C$. pneumoniae-specific PCR and analysis of the amplified samples as performed as described by Campbell et al. [9]. C. psittaci CF antigens were amplified with a PCR that amplifies both C. pneumoniae and C. psittaci, as described by Tong et al. [10]. PCR products $(40 \mu 1)$ were analysed by electrophoresis on an agarose $2 \%$ gel stained with ethidium bromide; $20 \%$ of the PCR reaction tubes were used for negative controls. All pipetting was done with aerosol-resistant tips and the recommendations of Kwok et al. were used to prevent carryover contamination [11].

\section{Mycoplasma hybridisation assay}

The GenProbe Mycoplasma tissue culture hybridisation assay (GenProbe Inc., San Diego, CA, USA) was used according to the manufacturer's instructions.

\section{DNA sequencing and phylogenetic tree construction}

The 373 DNA Sequencing System (Perkin Elmer, Foster City, USA) was used to sequence the amplified fragments according to the manufacturer's protocol. Sequence homology searches were done by use of the European Molecular Biology Laboratory nucleotide sequence database (EMBL; Heidelberg, Germany) and the GenBank nucleic acid database (National Center for Biotechnology Information, Bethesda, MD, USA). The GenBank nucleic acid database was used for the sequence retrieval of other frequent contaminants of tissue cultures and other bacteria. The CLUSTAL W program (EMBL), release 1.5, was used to calculate the homology of the sequences and to construct multiple alignments of the sequences [12]. The PHYLIP program (Phylogeny Inference Package; University of Washington, Seattle, USA), version $3.5 \mathrm{c}$, was used to construct a phylogenetic tree [13]. The maximum likelihood method was used to estimate the phylogenetic distances between the sequences [14].

\section{Serology}

A MIF assay with purified C. pneumoniae antigen from the WRF as described before was used to measure $C$. pneumoniae-specific IgG, IgM and IgA antibodies [3]. In addition, a commercially available MIF assay was used to compare $C$. pneumoniae-specific $\operatorname{IgG}$, IgM and IgA antibody titres (Labsystems OY). Sera were obtained from patients with clinical and serological evidence of recent respiratory tract infection due to $M$. pneumoniae $(\mathrm{n}=32)$ or $C$. pneumoniae $(\mathrm{n}=156)$.

\section{Results}

Four batches of $C$. pneumoniae antigen from the WRF were analysed. Lot numbers 7 and two batches of 17, which were provided in 1992, 1994 and 1995, respectively, were all mycoplasma PCR positive. Lot number 6, which was provided in 1991, was mycoplasma PCR negative. The microscopy slides from MRL for the detection of chlamydial antibodies were also positive for mycoplasma, as determined by PCR, while the slides from Labsystems for the detection of chlamydial antibodies and the $C$. psittaci $\mathrm{CF}$ antigen products were mycoplasma PCR negative. The specificity of the amplified products was confirmed by hybridisation with the internal probe GPO-2. Detectable chlamydial DNA was present in all samples, as determined by chlamydia-specific PCR, while results with all negative controls remained negative. All mycoplasma PCR positive chlamydia antigen samples were confirmed by the GenProbe Mycoplasma hybridisation assay. However, a total of five MRL slides were needed to obtain a weak positive signal in this hybridisation assay.

A DNA fragment of $280 \mathrm{bp}$ was PCR amplified with the primers GPO-3 and MGSO. After sequencing the product in both directions, a consensus sequence was constructed. The length of the remaining sequence was 200 nucleotides. This fragment contained the two variable regions $\mathrm{V} 3$ and $\mathrm{V} 5$ of the $16 \mathrm{~S}$ rRNA gene. Comparison of the nucleic acid sequences of all mycoplasma-positive $C$. pneumoniae antigen lot numbers of the WRF, including the mycoplasma-positive C. pneumoniae antigen slides from MRL Diagnostics, showed a high degree of similarity (Table 1).

The nucleic acid sequences of the Mycoplasma sp. found in the WRF and MRL products were further aligned with those of a group of nine known Mycoplasma spp. - M. hyosynoviae, M. hyopneumoniae, $M$. hominis, $M$. orale, $M$. arginini, $M$. fermentans, $M$. pneumoniae, $M$. genitalium and Acholeplasma laidlawii. Six other $16 \mathrm{~S}$ rRNA sequences were also aligned $-C$. pneumoniae, C. psittaci, C. trachomatis, Staphylococcus aureus, S. epidermidis and Escherichia coli. A phylogenetic tree was constructed to investigate the relationships among the group Mycoplasma spp. found in the WRF antigen, the mycoplasma found 


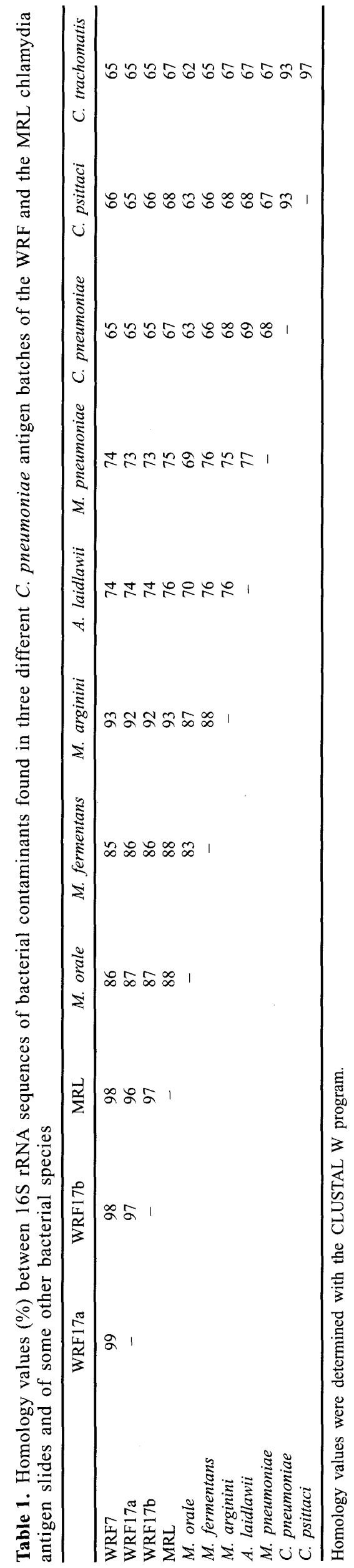


in the MRL antigen slides and other bacteria. Analysis of the phylogenetic tree revealed that the mycoplasma sequences from the WRF and MRL antigen products were very similar to those published for $M$. arginini (Fig. 1).

Serum samples (188) were used to determine the impact of mycoplasma contamination of these preparations on their use in serology. No fluorescence pattern was found compatible with cross-reactivity in the $C$. pneumoniae MIF assay when 32 serum samples from patients with recent $M$. pneumoniae infection were used. Also, no significant differences were observed in the $C$. pneumoniae-specific $\mathrm{IgG}$, IgM and $\operatorname{IgA}$ titres when the contaminated and clean $C$. pneumoniae antigens were used and their results were compared (data not shown).

Another confounding factor studied was the possibility that such mycoplasma contamination could alter the expression of antigens in the $C$. pneumoniae membrane. MIF results from patients with recent $C$. pneumoniae infection were compared with the contaminated and clean $C$. pneumoniae antigens; 156 serum samples were compared. Again, no significant difference was found in C. pneumoniae-specific IgG, $\operatorname{IgM}$ and $\operatorname{Ig} A$ titres (data not shown).

\section{Discussion}

The class Mollicutes consists of a very large group of prokaryotes distinguished phenotypically from other bacteria by their lack of cell walls. The largest group is formed by the genus Mycoplasma, of which $>90$ species have been described. Because of their small size $(300-800 \mathrm{~nm})$ and flexibility, mycoplasma can pass easily through 220 - and 450-nm filters used in cell culture laboratories [15]. Cell cultures are widely used in both diagnostic tests and in biomedical research settings, as well as in the production of antigens and vaccines by industry. Approximately $15 \%$ of all continuous cell lines are estimated to be contaminated with mycoplasmas worldwide [4]. At present, five species account for $>95 \%$ of these infections. The most prevalent contaminants are two human strains, $M$. orale and $M$. fermentans, two bovine Mollicutes, $M$. arginini and $A$. laidlawii and a porcine Mollicute, $M$. hyorhinis [16]. Mycoplasmal infection may induce various degrees of cytopathic effects (CPE) in cells. However, infection often remains inapparent. These inapparent infections may result in unreliable experiments as seen in the study by Tan et al. [17]. The operon described in their study has been found to be of mycoplasmal origin and not of chlamydial origin as initially reported. Also, mycoplasma infection can be

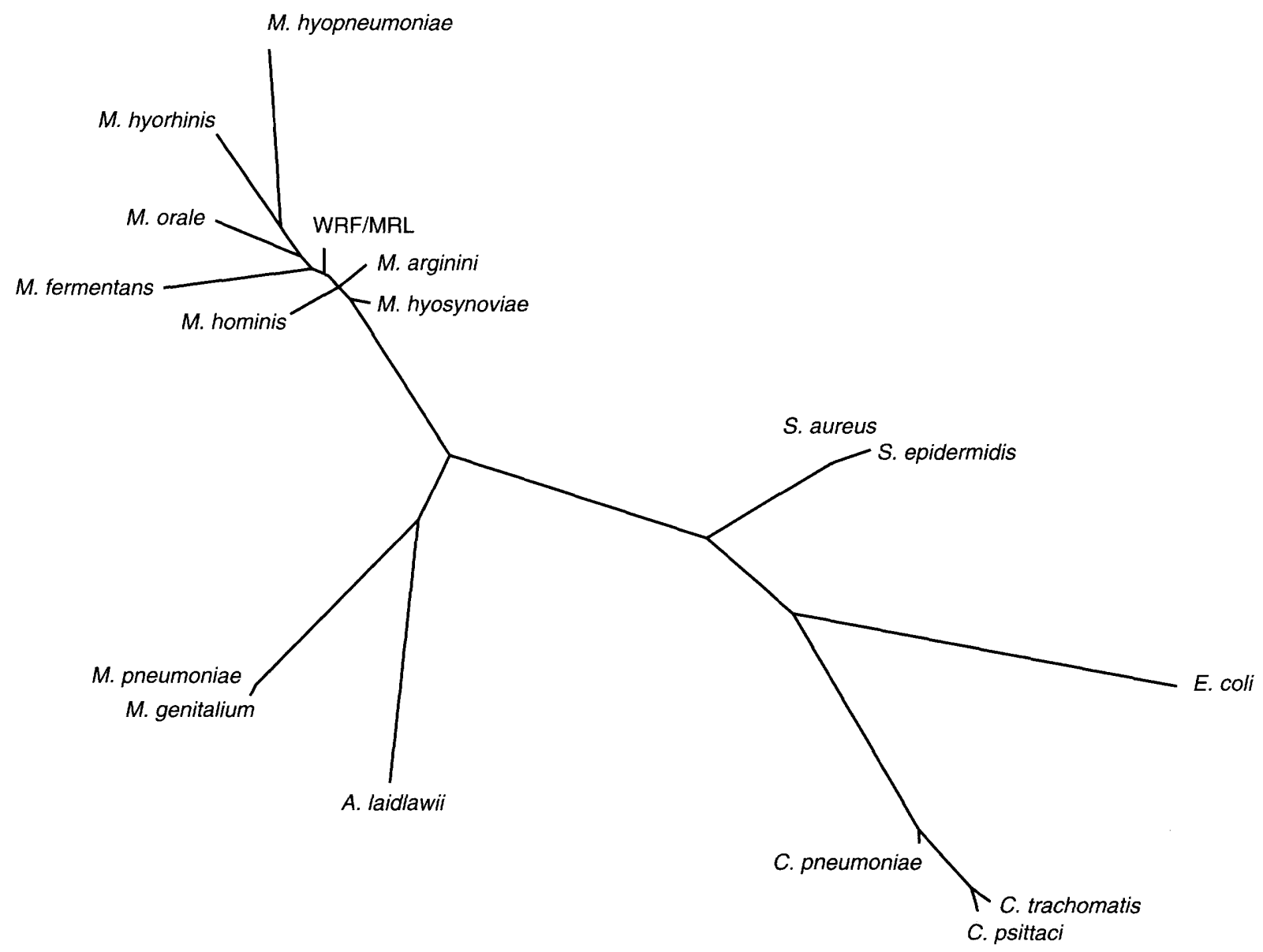

Fig. 1. Phylogenetic tree of the WRF and MRL mycoplasma contaminant and other bacteria based on 16S rRNA sequences. The phylogenetic tree was calculated by the maximum likelihood procedure of the PHYLIP program. 
responsible for the distribution of unsafe biological products, including vaccines and antigen preparations for serological diagnosis $[18,19]$.

Serological diagnosis of chlamydia infections is often based on CF or MIF results [1,3]. The antigens used in these tests are commonly purified from chlamydiainfected cell lines. Messmer et al. reported that mycoplasma contamination of $C$. pneumoniae isolated from clinical specimens often occurs [4]. Their findings suggest that the C. pneumoniae isolates were contaminated by isolation of respiratory specimens, or by propagation in contaminated cell lines.

In the present study, mycoplasma contamination was observed in commercially available antigens marketed for the detection of chlamydia-specific antibodies. Several lot numbers of the purified $C$. pneumoniae antigen from the WRF were contaminated with identical mycoplasmas, as determined by PCR and sequence analysis. Also, the MRL antigen slides for the detection of chlamydial antibodies were PCR positive for the same mycoplasma. One of the explanations for this result can be found in the sharing of cell lines or the use of the same organism for the production of the antigen, or both. Both companies use the AR-39 organism and HeLa 229 cell line for the production of the $C$. pneumoniae antigen. Of interest, the organism used by MRL was provided by the WRF.

Analysis of the phylogenetic tree revealed that the mycoplasma sequences from the WRF and MRL antigen products were closely related, but not identical, to $M$. arginini. This indicates the possibility of a new Mycoplasma species, which has not previously been submitted to the GenBank and EMBL databases.

Several methods were used by MRL Diagnostics, Labsystems and the WRF to detect mycoplasmal contamination before culture of the chlamydial antigens. Mycoplasma detection at MRL diagnostics was done by the GenProbe mycoplasma screening assay (Dr W. Hogrefe, personal communication). Failure to detect mycoplasmal contamination by hybridisation assay was probably due to the small number of organisms present in the samples, as seen in the present study. For every new chlamydia antigen lot the cell culture system from Labsystems was tested with fluorochromic dye (DAPI). Also, the cells were tested twice a year by the mycoplasma PCR ELISA from Boehringer Mannheim (Dr M. Ristola, personal communication). The WRF tested only the stock HeLa 229 cells by DAPI staining twice a year (Dr C. C. Kuo, personal communication). Despite these prevention strategies, contamination with mycoplasma has apparently occurred frequently. These results demonstrate that the PCR may currently be the preferred tool for the detection of mycoplasma contamination in cell cultures and chlamydial strains. Although this particular mycoplasma contamination had no significant impact on serodiagnosis of $C$. pneumoniae infection by MIF, caution should be exercised. Inapparent cell line contamination with human Mycoplasma spp. is always possible, which can have a major impact on the serodiagnosis of many bacterial and viral infections, and may confound research efforts.

In conclusion, mycoplasma contamination of commercially available antigens for the serodiagnosis of chlamydia infections has occurred, and therefore routine screening of all antigen preparations obtained by tissue culture by sensitive techniques is recommended before their distribution and use.

This work was presented in part at the 36 th Interscience Conference on Antimicrobial Agents and Chemotherapy, New Orleans, USA, 15-18 Sept. 1996, and the 3rd meeting of the European Society for Chlamydia Research, Vienna, Austria, 11-14 Sept. 1996.

\section{References}

1. Wong KH, Skelton SK, Daugharty H. Utility of complement fixation and microimmunofluorescence assays for detecting serologic responses in patients with clinically diagnosed psittacosis. J Clin Microbiol 1994; 32: 2417-2421.

2. Ossewaarde JM, de Vries A, van den Hoek JA, van Loon AM. Enzyme immunoassay with enhanced specificity for detection of antibodies to Chlamydia trachomatis. $J$ Clin Microbiol 1994; 32: 1419-1426.

3. Verkooyen RP, Hazenberg MA, Van Haaren GH et al. Agerelated interference with Chlamydia pneumoniae microimmunofluorescence serology due to circulating rheumatoid factor. J Clin Microbiol 1992; 30: 1287-1290.

4. Messmer TO, Black CM, Thacker WL. Mycoplasma contamination of chlamydiae isolated from clinical specimens. APMIS 1994; 102: 793-796.

5. Gilroy CB, Thomas BJ, Taylor-Robinson D. Small numbers of Chlamydia trachomatis elementary bodies on slides detected by the polymerase chain reaction. J Clin Pathol 1992; 45: $531-532$.

6. Boom R, Sol CJA, Salimans MMM, Jansen CL, Wertheim-van Dillen PME, van der Noordaa J. Rapid and simple method for purification of nucleic acids. J Clin Microbiol 1990; 28: $495-503$.

7. van Kuppeveld FJ, Johansson KE, Galama JM et al. Detection of mycoplasma contamination in cell cultures by a mycoplasma group-specific PCR. Appl Environ Microbiol 1994; 60: $149-152$.

8. van Kuppeveld FJ, van der Logt JTM, Angulo AF et al. Genus- and species-specific identification of mycoplasmas by 16S rRNA amplification. Appl Environ Microbiol 1992; 58: 2606-2615.

9. Campbell LA, Perez Melgosa M, Hamilton DJ, Kuo C-C, Grayston JT. Detection of Chlamydia pneumoniae by polymerase chain reaction. J Clin Microbiol 1992; 30: 434-439.

10. Tong CY, Sillis M. Detection of Chlamydia pneumoniae and Chlamydia psittaci in sputum samples by PCR. $J$ Clin Pathol 1993; 46: 313-317.

11. Kwok SK, Higuchi R. Avoiding false positives with PCR. Nature 1989; 339: 237-238.

12. Thompson JD, Higgins DG, Gibson TJ. ClustAL W: improving the sensitivity of progressive multiple sequence alignment through sequence weighting, position-specific gap penalties and weight matrix choice. Nucleic Acids Res 1994; 22: $4673-4680$.

13. Sanderson MJ. Flexible phylogeny reconstruction: a review of phylogenetic inference packages using parsimony. Systematic Zoology 1990; 39: 414-420.

14. Felsenstein J. Evolutionary trees from DNA sequences: a 
maximum likelihood approach. $J$ Mol Evol 1981; 17: 368-376.

15. Spaepen M, Angulo AF, Marynen P, Cassiman JJ. Detection of bacterial and mycoplasma contamination in cell cultures by polymerase chain reaction. FEMS Microbiol Lett 1992; 78: 89-94.

16. Teyssou R, Poutiers F, Saillard C et al. Detection of mollicute contamination in cell cultures by $16 \mathrm{~S}$ rDNA amplification. $\mathrm{Mol}$ Cell Probes 1993; 7: 209-216.
17. Tan M, Klein R, Grant R, Ganem D, Engel J. Cloning and characterization of the RNA polymerase alpha-subunit operon of Chlamydia trachomatis. J Bacteriol 1993; 175: 7150-7159.

18. Stanbridge E. Mycoplasmas and cell cultures. Bacteriol Rev 1971; 35: 206-227.

19. Harper DR, Kangro HO, Argent S, Heath RB. Reduction in immunoreactivity of varicella-zoster virus proteins induced by mycoplasma contamination. J Virol Methods 1988; 20: 65-72. 